\title{
Effect of Ultra-Turrax on Nanocellulose Produced by Acid Hydrolysis and Modified by Nano ZnO by Sol-Gel Method
}

\author{
Amanda Ramos Aragão Meloㅁ, Jose Carlos Dutra Filho², Roberto Pinto Cucinelli Neto', \\ Wellington Silva Ferreira3 ${ }^{3}$, Bráulio Soares Archanjo4, Raphael Verdan Curti4, \\ Maria Ines Bruno Tavares ${ }^{1}$
}

\begin{abstract}
${ }^{1}$ Instituto de Macromoléculas Professora Eloisa Mano, Universidade Federal do Rio de Janeiro, Bl J do Centro de Tecnologia, Ilh do Fundão, Rio de Janeiro, RJ, Brasil

${ }^{2}$ Instituto SENAI de Inovação em Biossintéticos e Fibras, Universidade Federal do Rio de Janeiro, Rio de Janeiro, Brasil

${ }^{3}$ Instituto de Biofísica Carlos Chagas, Universidade Federal do Rio de Janeiro, Rio de Janeiro, Brasil

${ }^{4}$ Divisão de Metrologia de Materiais, Instituto Nacional de Metrologia, Qualidade e Tecnologia (INMETRO), Duque de Caxias, Brasil

Email: mibt@ima.ufrj.br
\end{abstract}

How to cite this paper: Melo, A.R.A., Filho, J.C.D., Neto, R.P.C., Ferreira, W.S., Archanjo, B.S., Curti, R.V. and Tavares, M.I.B. (2020) Effect of Ultra-Turrax on Nanocellulose Produced by Acid Hydrolysis and Modified by Nano ZnO by Sol-Gel Method. Materials Sciences and Applications, 11, 150-166

https://doi.org/10.4236/msa.2020.112009

Received: November 23, 2019

Accepted: February 9, 2020

Published: February 12, 2020

Copyright $\odot 2020$ by author(s) and Scientific Research Publishing Inc. This work is licensed under the Creative Commons Attribution International License (CC BY 4.0).

http://creativecommons.org/licenses/by/4.0/

\begin{abstract}
Cellulose nanocrystals (NCC) and cellulose nanofibrils (CNF) were obtained by a single step process, with synergy between $64 \%$ sulfuric acid hydrolysis and high shear from ultra-turrax stirring, which is an advantageous process for disintegrating cellulose microcrystalline and also may improve the hydrolysis process. The surface modification on the cellulose was performed by the sol-gel process, in which the sulfate groups from hydrolysis were replaced by nanoparticles of zinc oxide, which led to the increase of up to $54 \mathrm{C}$ Tonset, according to thermogravimetric analysis (TGA) results. The morphology and crystallinity degree were characterized by Helium Ion Microscopy (HIM), atomic force microscopy (AFM) and X-ray diffraction. In addition, the $\mathrm{ZnO}$ band was observed in Fourier transform infrared spectroscopy, furthermore, the change in the zeta potential confirmed the cellulose modification. The changes in the values of proton spin-spin relaxation time for the systems showing the confined hydrogen in the rigid domains, confirmed the results observed with the aforementioned techniques, for both cellulose after hydrolysis and $\mathrm{ZnO}$ modified cellulose, suggesting that $\mathrm{ZnO}$ disrupted crystal formation in cellulose.
\end{abstract}

\section{Keywords}

Cellulose Nanocrystal, Cellulose Nanofibril, Nano Zinc Oxide, Acid Hydrolysis, Sol-Gel 


\section{Introduction}

Cellulose has applications in several areas due to its unique properties that are compatible with the growing search for materials from renewable sources. It is a biopolymer that is the main constituent of the cell wall of plants, and its extraction process follows the top-down strategy and can be mechanically or chemically extracted. Cellulose nanocrystals (NCC) are one of the materials obtained from this polymer, which can be used for several studies due to its versatility and characteristics. So, many studies report the obtaining cellulose nanocrystals (NCC) by hydrolysis with strong mineral acids as the most commonly used methodology, such as formic acid, acetic acid, sulfuric acid, hydrochloric acid, phosphoric acid or acid mixtures [1]-[5]; in 2012, Espinosa [6] investigated the influence of the three later acids behaved on the cellulose hydrolysis, as well as the influence on the dispersion of NCCs in different solvents and the differences in thermal stability. It was observed that NCCs obtained from hydrochloric acid treatment, presented higher thermal stability when compared to the ones obtained from the other acids, which was attributed to the absence of residual sulfates and phosphate groups.

Some issues are attributed to acid hydrolysis process, such as the recovery of the used acid, large amounts of base employed for neutralization, large amount of water, equipment corrosion and environmental pollution [7] [8]. Alternatively, other reagents such as phosphotungstic acid [9] [10] and dicarboxylic acids are used, which are more easily recoverable by the cold crystallization process and still have enough solubility at elevated temperatures, being able to hydrolyze the amorphous part of the cellulose and thus, be able to produce NCCs [7]. Another major advantage related to the use of these dicarboxylic acids is the possibility of cellulose surface modification through the Fischer-Speier Esterification reaction, as reported by [8] [11] [12] [13] [14].

Many works in the literature describe that the hydrolysis of cellulose with sulfuric acid can be found; where concentration, time and temperature of the reaction are cited as fundamental factors for the successful of the process. The break of glycosidic bonds in cellulose occurs preferentially in the amorphous region, while the crystalline part is less affected [15]. Wang and co-workers [16] studied the kinetics of acid hydrolysis and classified it in three stages: the first one occurred in the amorphous region, which promoted depolymerization of cellulose fiber; in the second one, they observed the glucose [4] [8] [11] [14] formation from cellulose, needing more severe hydrolysis conditions; the third one directly converts cellulose to monosaccharides.

Sulfuric acid concentration and temperature were crucial in reducing the degree of polymerization (DP) of cellulose and yield of NCC, since with $16 \mathrm{wt} \%$ acid, the DP decreases from 700 to 200 along temperature increase from $45^{\circ} \mathrm{C}$ to $85^{\circ} \mathrm{C}$, maintaining the yield; by increasing the acid concentration to $64 \%$ the DP was not altered, but the yield decreased due to competition between depolymerization and degradation reactions [16] [17]. Wang's group [18] optimized this 
process by balancing the yield of NCC and the percentage of cellulosic solid residue (CSR) generated, and this solid was transformed into NCC by the addition of one additional reaction step: the mechanical defibration stage. Although $60 \%$ acid concentration provided a higher percentage of NCC [18] [19], Chen's group [20] found that $58 \mathrm{wt} \%$ acid concentration was the transition point for the highest yield of NCC, despite of the higher lengths achieved.

NCCs and cellulose nanofibrils (CNFs) have interesting properties beyond their mechanical reinforcement to nanocomposites; those properties are related to the numerous possibilities of chemical modifications over hydroxyls located at cellulose surface, which can improve the polymer-cellulose interaction, decreasing the tendency for agglomeration and can add new functionality to cellulose for different applications such as: biomedical engineering, electronics and energy and water treatment sectors [1] [21]. Among the many modifications, the anchoring of inorganic particles in cellulose has been investigated to confer antimicrobial, anti-UV and flame retardant properties, as in textiles, and to extend the thermal stability range, which allows to process those materials at higher temperatures [1] [22] [23]. Due to its good thermal stability and biocidal property, zinc oxide is a promising material, besides having a wide absorption range of UV radiation in the $260-370 \mathrm{~nm}$ range [22] [24] it possesses good photo stability, low toxicity and biocompatibility, which makes it an interesting material for packaging and medical area [25] [26]. Among the methodologies for obtaining inorganic nanoparticles, the sol-gel methodology is advantageous because it is a low-cost process, with good control over the parameters and reaction occurs at lower temperatures when compared to other methodologies. Therefore, zinc oxide can be obtained in situ from a chemical precursor undergoing hydrolysis and condensation reaction. These reactions are controlled by the $\mathrm{pH}$ and temperature of the heat treatment performed at the end of the reaction [27] [28] [29] [30].

Much research is oriented to increase the yield of NCCs, reduce cellulose decomposition by monitoring the production of NCC and cellulosic solid residues (CSR) obtained from acid hydrolysis processes [17] [18]; other efforts are focused in reusing cellulosic tailings from the final bleached kraft pulp process [19]. The CSR is the precipitate obtained after the centrifugation at the end of the hydrolysis reaction, which being submitted to another mechanical step can render NCC, reducing the amount of waste generated, according to Wang [18]. Normally the isolation process could be improved by ultrasonication [14], ultra-turrax [31], the mechanical process by ball mill equipped [10] as well as combine ultrasonication and ultra-turrax showed better results when used simultaneous [8]. The purpose of this work was to perform the acid hydrolysis of microcrystalline cellulose in a single step, in order to achieve that goal, the mechanical stirrer (ultra-turrax) was used before and after the reaction. The chemical modification with zinc oxide is expected to improve the thermal stability of the cellulose and to confer photocatalytic activity to the composite. 


\section{Experimental}

\subsection{Materials and Reagents}

Microcrystalline cellulose (MCC) was purchased from Synth (density 0.26 - 0.32 $\mathrm{g} / \mathrm{cm}^{3}$, polymerization grade 350 ), nanocrystalline cellulose was purchased from Celluforce (density $0.7 \mathrm{~g} / \mathrm{cm}^{3}$ ) (NCC_C), sulfuric acid, sodium hydroxide, ethyl alcohol and di-hydrated zinc acetate were obtained from Sigma-Aldrich. The reagents were used as received.

\subsection{Microcrystalline Cellulose Acid Hydrolysis}

Cellulose nanocrystals were obtained from acid hydrolisis of microcrystalline cellulose. First, a suspension of MCC $\left(10 \%{ }^{\mathrm{w} / \mathrm{v}}\right)$ with $32 \%{ }^{\mathrm{v} / \mathrm{v}}$ sulfuric acid was produced and homogenized on a mechanical stirrer (IKA RW20) for $30 \mathrm{~min}$ at $17,000 \mathrm{rpm}$. Then the acid concentration was increased up to $64 \%^{\mathrm{v} / \mathrm{v}}$, and hydrolysis continued for a further $2 \mathrm{~h}$ under reflux at $50^{\circ} \mathrm{C}$. During the addition of sulfuric acid, the system was cooled with a cold ice bath.

By the end of the reaction time, $300 \mathrm{ml}$ of cold water was added to stop the hydrolysis reaction, after which, the suspension (identified as NCC_H) was taken to the mechanical stirrer for a further $20 \mathrm{~min}$ at 17,000 rpm. Finally, dialysis (molecular weight cut-off 14,000) was performed to neutralize the acid suspension, and the suspension stored in a refrigerator for approximately $24 \mathrm{~h}$.

\subsection{Sol-Gel Reaction at Cellulose Surface}

The previously obtained cellulose suspension $(200 \mathrm{ml})$ was submitted to reaction with the same volume of ethanol, adjusting the $\mathrm{pH}$ to 8 with sodium hydroxide solution (5 M).

The metal precursor used was zinc acetate dihydrate, which, being previously dissolved in ethanol for $24 \mathrm{~h}$, reacted with cellulose in a 1:1 ratio. The solution containing the precursor was dropped into the cellulose suspension, while $\mathrm{pH}$ was monitored and corrected to $\mathrm{pH} 8$. After the addition was complete, the reaction was heated at $80 \mathrm{C}$ under reflux for $2 \mathrm{~h}$. Then, part of the solvent was removed by evaporation, the precipitate washed with ethanol and centrifuged ( $4 \mathrm{x}$ at $3500 \mathrm{rpm}$ for $10 \mathrm{~min}$ ). At each washing step the cellulose was re-suspended with the aid of tip ultrasonic homogenizer (Ultronique QR500, frequency of 20 $\mathrm{KHz}$ ). The sample was oven dried at $120^{\circ} \mathrm{C}$ for $1 \mathrm{~h} \mathrm{[25].}$

\subsection{Characterizations}

\subsubsection{Zeta Potential}

The Zeta potential of the samples was measured on the NICOMP DLS/ZLS, model Nano Z3000, by the Electrophoretic light scattering technique. Concentration suspensions of $0.001 \mathrm{wt} \%$ were prepared, which before being analyzed were dispersed for $1 \mathrm{~min}$ in Ultrasonic QR500 ultrasound, then tripled analyzed at room temperature. 


\subsubsection{Scanning Helium Ion Microscope}

Helium beam assisted electron microscopy images were acquired on the Zeiss Orion nanofab model equipped with tungsten filament, operating at a constant voltage of $30 \mathrm{kV}$. The sample preparation involved suspension of $0.5 \mathrm{mg}$ cellulose in $1 \mathrm{ml}$ acetone, which was then diluted $50 \times$ and dispersed in ultrasonic bath for $15 \mathrm{~min}$. Then a drop was deposited on the silicon substrate, and the samples analyzed. As these are insulating samples, floodgun was used to neutralize the surface charge.

\subsubsection{Atomic Force Microscope}

Cellulose samples were prepared in aqueous suspensions $\left(1 \%^{\mathrm{w} / v}\right)$, these suspensions were diluted $100 \times$ and sonicated for $15 \mathrm{~min}$. A drop of the diluted suspension was deposited on the surface of the freshly cleaved mica, then dried under $\mathrm{N}_{2}$ flux. Images were acquired on a Dimension Icon ${ }^{\circledast}$ (Bruker, Santa Barbara-CA) AFM instrument using Peak Force Tapping QNM scan mode, cantilever model HQ-300-AU (Oxford Instruments ${ }^{\circledR}$, UK), spring constant $40 \mathrm{~N} / \mathrm{m}$, average radius of $10 \mathrm{~nm}$ and $300 \mathrm{kHz}$ frequency.

\subsubsection{X-Ray Diffraction}

A Rigaku Ultima IV X-ray diffractometer, operating with voltage and current of $40 \mathrm{Kv}$ and $30 \mathrm{~mA}$, respectively, was used for X-ray diffraction characterization. $\mathrm{X}$-rays were obtained from a CuK $\alpha$ source $(\kappa=1.5418 \AA)$. The analysis conditions for the MCC, NCC_C and NCC_H samples were made at $0.01^{\circ} / \mathrm{s}, 2 \theta$ between $10^{\circ}$ and $40^{\circ}$, with $2 \theta$ for the NCC-Zn sample between $10^{\circ}$ and $80^{\circ}$. The analyses were carried out at room temperature.

The size of the crystallite, $T$, perpendicular to the crystalline $\left(\begin{array}{lll}2 & 0 & 0\end{array}\right)$ plane, peak $2 \theta$ at $22.5^{\circ}$, was calculated by the Scherrer equation:

$$
T=\frac{K \lambda}{\beta \cos \theta}
$$

where $K$ is the correction value $0.9, \lambda$ is the wavelength of the $\mathrm{X}$-rays ( $1.54 \AA$ ), $\beta$ is the FWHM of the diffractogram peak (radians), and $\theta$ is the peak diffraction angle.

The crystallinity index, CrI, was calculated by the Segal Method whose equation is:

$$
\mathrm{CrI}=\frac{I_{t}-I_{a}}{I_{t}} \times 100
$$

where $I_{t}$ is the total intensity of peak $200\left(2 \theta\right.$ at $\left.22.5^{\circ}\right), I_{a}$ is the intensity of the amorphous contribution at $2 \theta=18.4^{\circ}$.

\subsubsection{Fourier Transform Infrared Spectroscopy (FTIR)}

Analyses were performed in a Nicolet 6700-FT-IR equipment, using $\mathrm{KBr}$ pellet, $4 \mathrm{~cm}^{-1}$ spectral resolution and 16 . Sample was dried for $48 \mathrm{~h}$ before analysis.

\subsubsection{Thermogravimetric Analysis (TGA)}

The thermal stability of the materials was analyzed on TA Instruments model 
TGA Q-50, under nitrogen atmosphere, heating rate of $10 \mathrm{C} / \mathrm{min}$ in the temperature range of $30 \mathrm{C}-700^{\circ} \mathrm{C}$.

\subsubsection{Nuclear Magnetic Resonance Time Domain (TD-NMR)}

Relaxation time measurements were performed on a MARAN Ultra NMR (Oxford Instruments) NMR spectrometer at $30^{\circ} \mathrm{C} \pm 22^{\circ} \mathrm{C}$ with $0.54 \mathrm{~T}(23 \mathrm{MHz}$ for $\left.{ }^{1} \mathrm{H}\right)$. The samples were dried for $48 \mathrm{~h}$ in a vacuum oven at $60^{\circ} \mathrm{C}$ and placed directly into the $18 \mathrm{~mm}$ diameter glass tube for analysis.

The Magic Sandwich Echo pulse sequence was used to acquire the fully refocused Free Induction Decay (MSE-FID). All relaxation curves were acquired 512 points spaced by 0.5 microseconds, with a repeating time of 1 second, 128 scans and $34 \mathrm{~dB}$ receiver gain.

The obtained signals were adjusted according to the following equation:

$$
F_{R} \exp \left[-\frac{1}{2}\left(\frac{t-\mu_{a}}{T_{2^{*} F R}}\right)^{2}\right] \cdot\left(\frac{\sin (2 \pi t)}{2 \pi t}\right)+F_{M} \exp \left[-\left(\frac{t}{T_{2^{*} F M}}\right)^{\beta_{M}}\right]+k
$$

where $F_{R}$, and $F_{M}$ are the fractions of the rigid (crystalline) and mobile (amorphous) regions, respectively. $T_{2^{*}}$ is the transverse relaxation time of one of these fractions, $v$ is a constant component of sine wave oscillation based on Van Vleck's second and fourth moments. $\beta_{M}$ corresponds to the Weibullian function exponents ranging from close to zero to $1 . \mu_{a}$ is centroid of the Abragamian and $k$ is the offset or baseline of the relaxation signal that compensates for the influence of background signal during nonlinear adjustment.

Considering $a=1 / T_{2^{*} F R}$ and $b=2 \pi v$, the mean value of the second dipolar coupling moment can be obtained through the equation:

$$
\left\langle M_{2}\right\rangle=a^{2}+\frac{1}{3} b^{2} .
$$

\section{Results and Discussion}

\subsection{Zeta Potential Measurement}

Despite being an important parameter to evaluate the stability of suspensions, this technique was employed in order to follow the influence of the surface modification influenced the cellulose surface charge. NCC_H presented a zeta potential around $-44 \mathrm{mV}$ and reached a value close to $0 \mathrm{mV}$ when modified with zinc. The value for NCC_H is consistent with the literature, since there is a direct relationship of this value with hydrolysis times [15]. The negative value for NCC_H is the result of the repulsion forces between the sulphate groups, promoting the good dispersion of these nanoparticles in aqueous suspension [4] [9] [12] [15]. The sol-gel reaction was performed with cellulose in cellulosic suspension, avoiding agglomerations resulting from the lyophilization process [24] [32] [33].

\subsection{Morphological Studies}

The morphology of the materials was examined by helium ion microscopy 
(HIM) and atomic force microscopy (AFM). Microcrystalline cellulose (Figure 1 (a)) had a rod-like shape, rough surface and irregularities, such as the presence of fibrils on smaller scales that pierced the cellulosic fibers (see Figure 1(a)), which may facilitate the accessibility of acid during hydrolysis [3]. After the MCC hydrolysis reaction (Figure 1(d)), cellulose tended to agglomerate in a round-shaped cluster with a thin layer around it. AFM micrograph (Figure 1(e)) isolated two cellulose nanocrystals of $100 \mathrm{~nm}$ length and $3 \mathrm{~nm}$ diameter, revealing that the experimental hydrolysis conditions were able to produce cellulose nanocrystals, and its morphology, size and diameter were similar to those found in the literature [18] [34]. Comparing the images of Figure 1(d) and Figure 1(b)
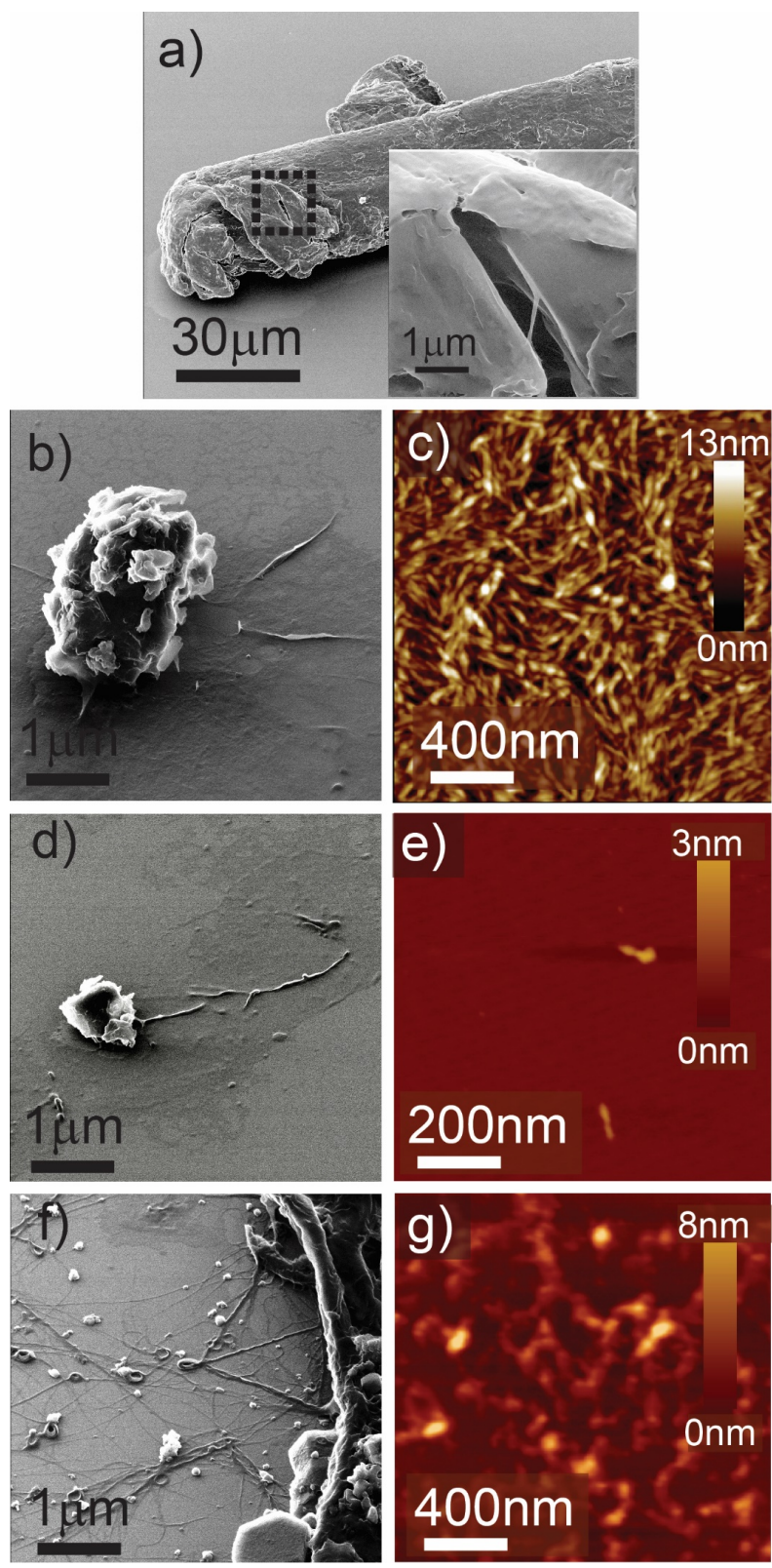

Figure 1. HIM images of MCC (a), NCC_C (b), NCC_H (d), and NCC_ZnO (f); topographic AFM image of sample NCC_C (c), NCC_H (e) and NCC_ZnO (g). 
(commercially obtained cellulose nanocrystals), both samples showed the same agglomeration tendency in round-shaped clusters and the presence of a thin layer around the cluster. The topography image for sample NCC_C (Figure $1(\mathrm{c})$ ), revealed that this clustering was formed from the network of randomly interconnected cellulose nanoparticles. This comparison between NCC_H and NCC_C permits to state that the methodology promotes the formation of cellulose nanocrystals, although agglomerated in micrometrical clusters.

HIM image Figure 1(f) confirms the presence of zinc nano oxide on the cellulose surface and in its non-associated form into reaction medium. It was observed that the zinc structure formed is porous and polydisperse (image not shown here), which may be related to the lack of control in the dropping of the metal precursor solution, $\mathrm{pH}$ fluctuations during the reaction and the absence of capping agent [27] [30] [35] [36]. Fibril-like structures were identified by microscopy, which correspond to the type of nanofibrillated cellulose. These structures are probably the result of partially hydrolyzed structures present in the CSR, which were defragmented in fibrils with higher aspect ratio by mechanical stirring of Ultra-turrax and the negative surface charges, which agrees with previous observations by [15] [18].

The AFM image (Figure 1(g)) complements the information in Figure 1(f), since the roughness on the cellulose surface as well as distinct morphologies are visible, which were attributed to the zinc oxide (round shape) anchored on the surface of the CNF.

\subsection{X-Ray Diffraction}

The X-ray diffraction pattern shown in Figure 2 corresponds to type I cellulose; all the samples have peaks at $2 \theta=15^{\circ}, 16^{\circ}, 21^{\circ}$ and $22.8^{\circ}$, such peaks correspond to the planes (110), (110), (012), and (200), respectively. The MCC and NCC_H samples exhibit a small $12.4^{\circ}$ shoulder that can be attributed to type II cellulose. Sèbe [34] confirmed the polymorphism of in NCCs (cellulose type I and II) obtained from hydrolysis with sulfuric acid, which is related to the concentration and time of acid addition. Jin's research group [32] also confirmed the coexistence of the two types of cellulose (I and II) by attributing a peak at $2 \theta$ equal to $12^{\circ}$ to type II pulp.

Gaussian deconvolution of the diffractograms allowed to better evaluate the profile of the crystalline planes before and after hydrolysis, making the changes of the crystallographic patterns more noticeable. There is a greater crystalline contribution to the MCC when compared to the NCC_H, if we consider the peak intensity at (110) and (200). The degree of crystallinity was calculated by the Segal method, which, as described by [37] is an efficient and widespread method in the literature. The data in Table 1 corroborate the abovementioned hypothesis, showing that the crystallinity is slightly lower for NCC_H. Therefore, although the mechanical effect benefited the disintegration of MCC the crystal structure of NCC_H has been preserved, acid hydrolysis under these 

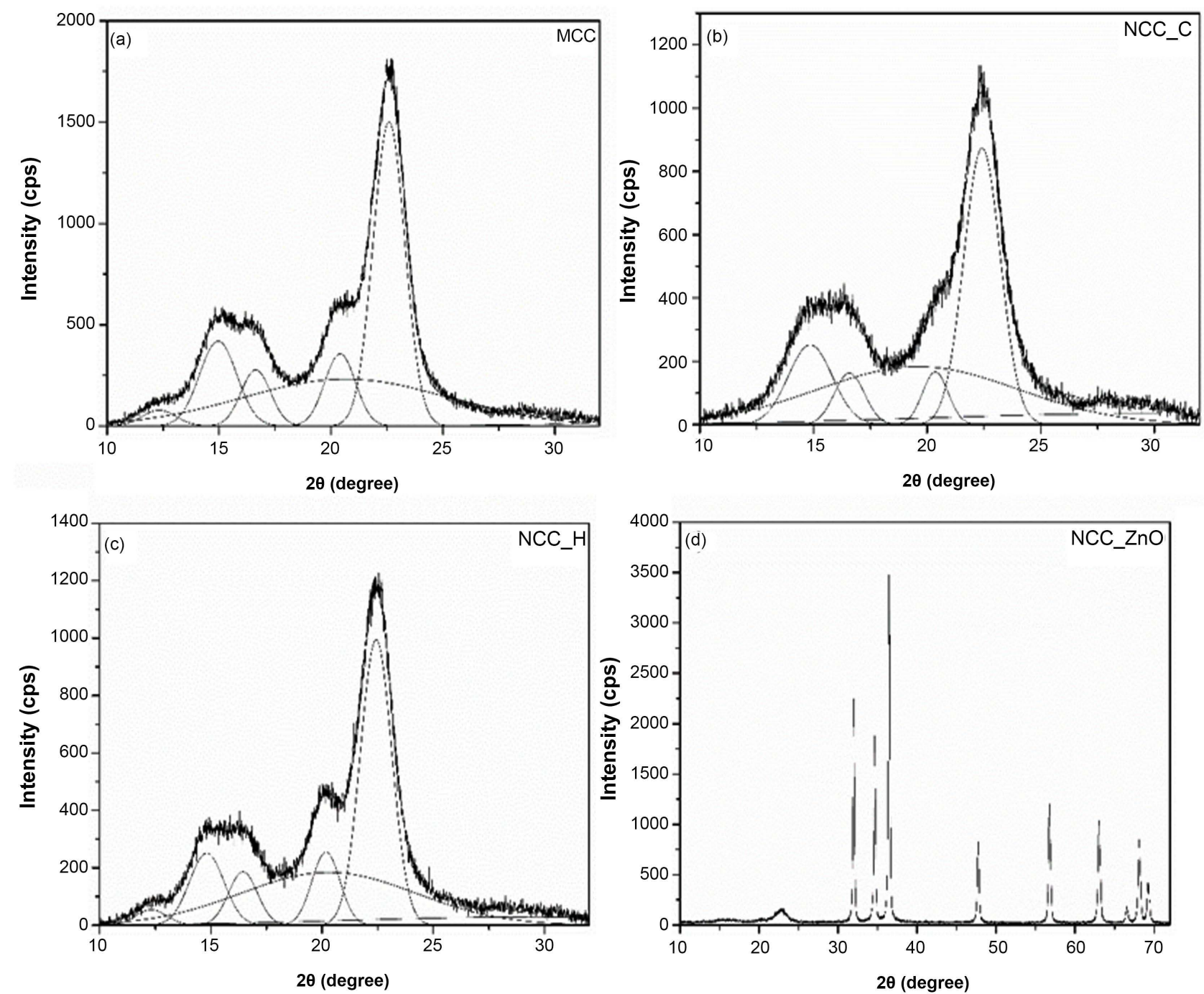

Figure 2. Diffractograms of MCC (a), NCC_C (b), NCC_H (c) and NCC_ZnO (d) samples. The deconvolution of the samples (a), (b) and (c) was performed, which evidenced the crystalline and amorphous contributions in the samples.

Table 1. Crystallinity index (CrI) and crystallite size (T200) data obtained from XRD.

\begin{tabular}{ccc}
\hline Samples & $T 200(\mathrm{~nm})$ & CrI (\%) \\
\hline MCC & 5.38 & 84 \\
NCC_C & 4.73 & 79 \\
NCC_H & 5.39 & 81 \\
\hline
\end{tabular}

conditions $\left(64 \%, 50^{\circ} \mathrm{C}, 120 \mathrm{~min}\right)$ favored degradation reactions, with cellulose being reduced to sugars or soluble by-products [2] [15] [16] [17] [31]. The divergence in the value of CrI and $T 200$ for NCC_H and NCC_C is at the origin of the fiber, since the DP varies from source to source, finally reflecting the length of the obtained cellulose crystal, as observed by Jia's group [12]. The values found for $T 200$ (Table 1), agree with report by Hamad [17], despite the fact of MCC and NCC_H values were identical. 
The well-defined diffraction peaks at $2 \theta=31.9^{\circ}(100), 34.7^{\circ}(002), 36.4^{\circ}(101)$, $47.7^{\circ}(102), 56.7^{\circ}(110), 63.1^{\circ}(103), 68.2^{\circ}(200)$ and $69.3^{\circ}$ (201) correspond to the hexagonal structure of the wurtzite, as shown in Figure 2(d) [22] [23] [24] [25] [35]. Still in the same Figure 2(d), the less intense diffraction peaks of cellulose are encountered, and it is possible to state that there is no presence of intermediate structures $(\varepsilon-\mathrm{Zn}(\mathrm{OH}) 2)$, as the ones specified by [28] [29].

No adjustments could be made for the NCC_ZnO sample due to the high zinc oxide diffraction signal.

\subsection{Fourier Transform Infrared Spectroscopy (FTIR)}

Changes in the chemical environment and the appearance of new absorption bands after chemical modifications were observed in the FTIR spectrum (Figure 3). The wide absorptions in the range of $3800-3000 \mathrm{~cm}^{-1}$ and in the region of $1638 \mathrm{~cm}^{-1}$ were attributed to the stretching and bending vibration of the $\mathrm{OH}$ groups present in the cellulose, even though it was dried [4] [15] [31]. The bands found in the range between $1420-897 \mathrm{~cm}^{-1}$ correspond to the chemical structure of cellulose, such as symmetric $\mathrm{CH}_{2}$ bonding, $\mathrm{C}-\mathrm{H}$ and $\mathrm{C}-\mathrm{O}$ vibration, $\mathrm{C}-\mathrm{O}-\mathrm{C}$ bridges, $\mathrm{C}-\mathrm{O}$ stretching, $\mathrm{C}-\mathrm{H}$ rocking and $\mathrm{C}-\mathrm{O}-\mathrm{C} \beta$-glycosidic bonding [15] [19] [38]. The emergence of the $540-412 \mathrm{~cm}^{-1} \mathrm{Zn}-\mathrm{O}$ bond broadband confirms that there are cellulose- $\mathrm{ZnO}$ structures, as suggested in Figure 1(e), and the characteristic cellulose bands are still present [22] [23] [25] [27] [35] [39].

Although negative zeta potential value was observed for NCC_H, cellulose-O- $\mathrm{SO}_{3}$ binding was not detected in the IR spectrum, therefore, the degree of sulfonation is below the FTIR detection limit [17].

\subsection{Thermogravimetric Analysis}

The degradation of MCC and NCC_C presents a single degradation pattern, as

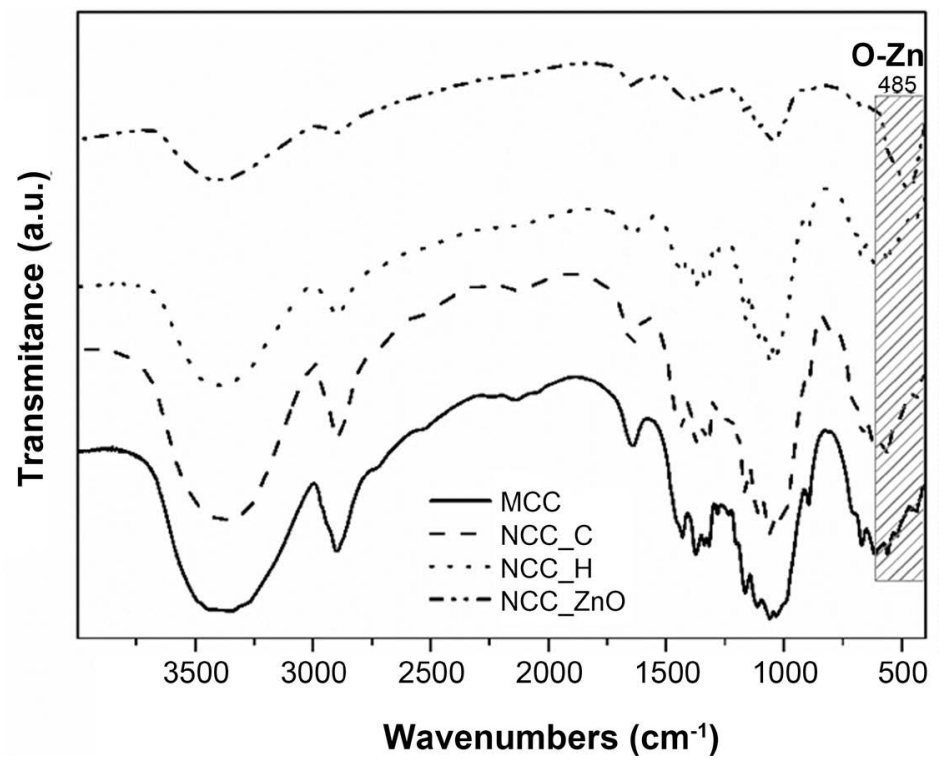

Figure 3. FTIR spectrum of MCC, NCC_C, NCC_H and NCC_ZnO samples. 
seen in Figure 4, corresponding to the steps of depolymerization, dehydration, decomposition of glycosidic units and ash formation, and such reactions occur concurrently [6] [12]. However, this profile is altered with the presence of sulphate groups or different surface functionalization, as described by [2] [5]. Obtaining cellulose nanocrystals by sulfuric acid hydrolysis, NCC_H, reduced the temperature at which $10 \%$ of the mass was degraded (T10\%, shown in Table 2) by up to $30 \%$ when compared to MCC. As observed in this paper, it is described in the literature that the presence of sulphate clusters on the cellulose surface reduces thermal stability, which agrees with Kargarzadesh group [15], which associated low thermal stability with longer hydrolysis time, making thus evident that there was a change in the degradation profile after the neutralization process, which possibly removed sulfate residues [2]. The NCC_C degraded in a single event, because as reported in the technical sheet, the neutralization of the groups with sodium hydroxide was performed.

In the DTG curve, it is possible to see the changes in the cellulose degradation behavior. The degradation of the NCC_H sample occurs over a wider temperature range, and events that occur at lower temperatures are related to the most accessible sulfated region, which, according to Kargarzadesh [15], corresponds

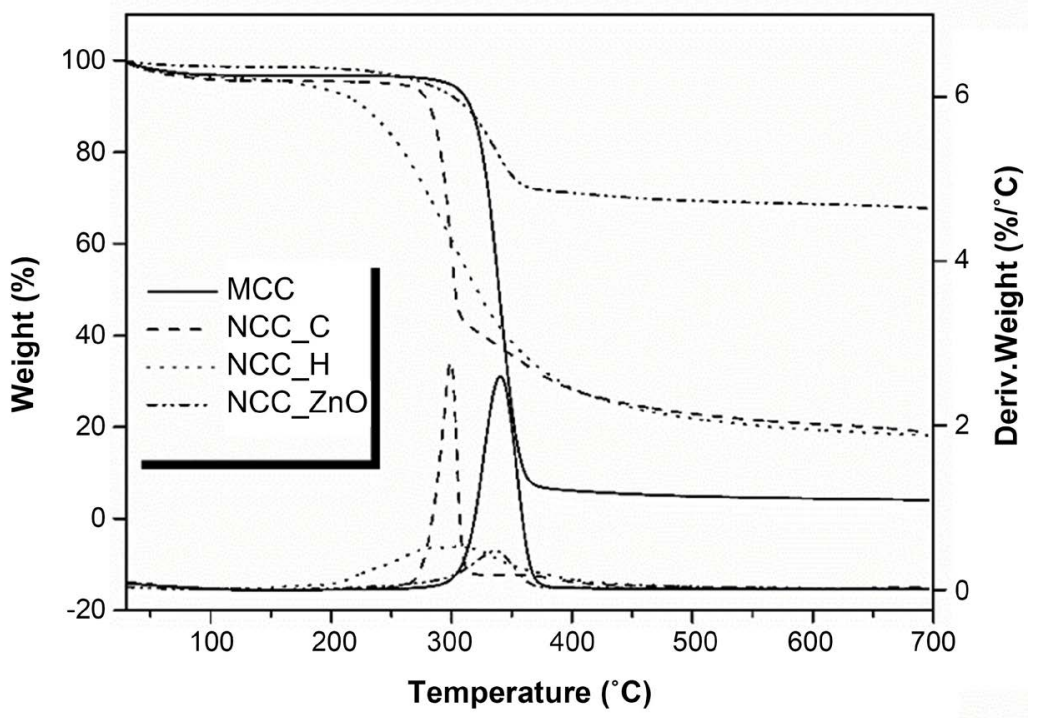

Figure 4. Mass loss curve and temperature mass loss derivative for MCC, NCC_C, NCC_H and NCC_ZnO cellulose samples.

Table 2. Data extracted from TGA curves, temperature relative to $10 \%$ mass loss (T10\%), peak temperature obtained from DTG, and \% residue of samples after analysis.

\begin{tabular}{cccc}
\hline Sample & $\mathrm{T} 10 \%\left({ }^{\circ} \mathrm{C}\right)$ & $\mathrm{T}_{\text {peak }}\left({ }^{\circ} \mathrm{C}\right)$ & \% Residue \\
\hline MCC & 316 & 340 & 4 \\
NCC_C & 282 & 300 & 19 \\
NCC_H & 226 & 300 & 18 \\
NCC_ZnO & 336 & 335 & 68
\end{tabular}


to the amorphous region; also, it was attributed to the increase in sulfated chain terminals, which begin to degrade faster [2] [6] [19]. The increase in residue percentage after hydrolysis with sulfuric acid compared to MCC is related to the flame-retardant property of sulfate groups [2] [6] [15].

Zinc oxide surface modification (NCC_ZnO) improved cellulose thermal stability compared to NCC_H, evidenced by a Tonset increase of 54C, as shown in Table 2. Increase on thermal stability of sulfuric acid hydrolysis produced cellulose was reported by Wang [2], who neutralized the acidic suspension with sodium hydroxide. The observed Tonset increase of NCC_ZnO is justified by the removal of these acid groups and the increase of zinc nano oxide on the surface. Comparing the DTG curves of NCC_H and NCC_ZnO samples, the peak width was decreased after modification, like MCC. Giraldi [39] attributed this narrowing of the DTG peak to the conversion of the zinc metal precursor to zinc oxide, where the acetate groups degraded under $200^{\circ} \mathrm{C}$. All samples showed a mass loss of up to $5 \%$ at $100^{\circ} \mathrm{C}$ and this loss can be attributed to humidity [15].

The NCC_ZnO sample exhibited $5 \%$ mass loss at $100^{\circ} \mathrm{C}$, followed by a single degradation event with $30 \%$ mass loss. The residue percentage corresponds to the sulfur residue, derived from the NCC_H sulfate ester groups, and to the modification with zinc oxide. Azizi's group [25] tested different proportions of precursor and cellulose, they found that the higher the amount of precursor used, the higher the percentage of waste generated, which agrees with [29]. Arputharaj [22] observed that the $\mathrm{ZnO}$ produced on the surface of the cotton cloth was able to increase its flame-retardant characteristics. Zinc oxide does not lose mass during the temperature range studied.

\subsection{Nuclear Magnetic Resonance Time Domain (TD-NMR)}

The nuclear magnetic relaxation technique allows the morphological investigation of nanostructured systems such as cellulose, through the relaxation times of protons present in each environment or domain, where the polymer segments assume a specific conformation/arrangement. From the measurements obtained by MSE-FID (Figure 5) and the adjustment of the points based on Equation (3), it is observed that all samples have two well defined domains, one of them rigid $\left(F_{r}\right)$ associated with the crystalline fraction of matrix, and another of higher molecular mobility $\left(F_{m}\right)$ related to the amorphous fraction of cellulose. The crystallinity degree values obtained from $F_{r}$ are like those found in CrI, calculated by XRD analysis. In Table 3, MCC and NCC_C have similar values of relaxation times, which may be associated with uniformity in particle size, as seen in Figure 1 and discussed above. For NCC_H these relaxation times are shorter since not only cellulose nanocrystals are present but also cellulose nanofibrils which have a highly oriented morphology. For the NCC_ZnO sample, it is observed that the presence of oxide in the cellulose structure reduces its crystalline fraction to $86 \%$ [40].

The mean value of the second dipole coupling moment $\left\langle M_{2}>\right.$ is associated to 

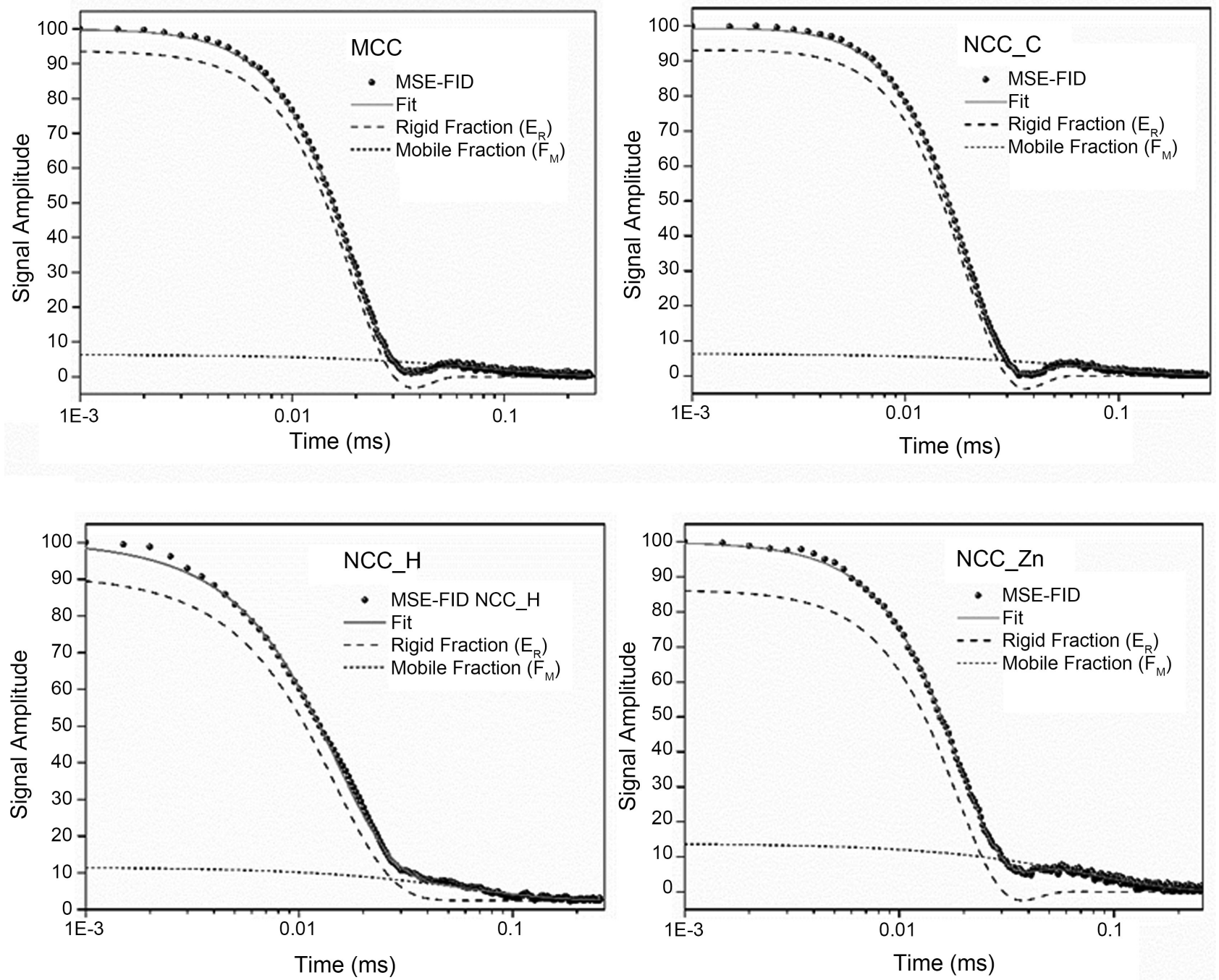

Figure 5. MSE-refocused FID curves with nonlinear fit based on Equation (3).

the distance between cellulose chain segments in the crystalline fraction, being proportional to the coupling force between the hydrogen nuclei in the crystals. The value of $9262 \mathrm{rad} \cdot \mathrm{ms}^{-2}$, shown in Table 3, found for NCC_H indicates that while having a slightly lower crystallinity than the MCC and NCC_C samples, the crystals in this matrix have a larger organization, with better packed segments and high coupling [41] [42]. However, for the NCC_ZnO sample, the value of $\left\langle M_{2}\right\rangle$ decreases to $5966 \mathrm{rad} \cdot \mathrm{ms}^{-2}$ indicating that, in addition to reducing the percentage of crystals, zinc oxide makes it difficult to rearrange the chains, providing more imperfect crystals.

\section{Conclusion}

Nanocrystals and cellulose nanofibrils were produced in a single step combining acid hydrolysis mechanical stirring with ultra-turrax, which was responsible for the improvement of MCC dispersibility in acid medium, the high shear promoted the mass transfer and helped the disintegrations and isolation to NCC 
Table 3. Transversal relaxation time for rigid and mobile fraction.

\begin{tabular}{cccccc}
\hline Sample & $T_{2^{*} F r}(\mu \mathrm{s})$ & $X c=F_{R}(\%)$ & $T_{2^{*} F m}(\mu \mathrm{s})$ & $F_{m}(\%)$ & $<M_{2}>\left(\mathrm{rad} \cdot \mathrm{ms}^{-2}\right)$ \\
\hline MCC & 20 & 94 & 84 & 6 & 6311 \\
NCC_C & 20 & 94 & 79 & 6 & 6311 \\
NCC_H & 10 & 91 & 91 & 9 & 9262 \\
NCC_ZnO & 20 & 86 & 75 & 14 & 5966 \\
\hline
\end{tabular}

and NFC. Next, the nanoparticles were superficially modified with nano oxide by sol-gel methodology under low temperature, as found by HIM and AFM, FTIR, XRD, TGA and NMR analyses. The NMR technique clarified the changes in the constituent fractions of cellulose, rigid and mobile fraction, validating the observations made through the XRD technique. Furthermore, the crystalline fraction of NCC_ZnO was detected by this technique. The properties presented by nanocellulose and zinc oxide were combined into a single particle, and the improvement in thermal stability enables the preparation of nanocomposite via melting mixture. Therefore, this nanoparticle is expected to play the reinforcing role and barrier property, characteristic of nanocellulose, and antimicrobial and anti-UV activity, property of $\mathrm{ZnO}$, when added to a polymer. According to all properties studied about this particle we can say that it could be used in packing.

\section{Acknowledgements}

This work was financially supported by CAPES code 001 and CNPQ. The authors thank for the Biological Physical Laboratory, located in Carlos Chagas Institute of Biophysics, special for the Professors Dr. Gilberto Weissmüller and Dr. Paulo Bish. We also thank for Inorganic Chemistry Department, Institute of Chemistry, mainly MSc. Glaucia Martins for all the support and contributions. Both Institutes are in the Federal University of Rio de Janeiro.

\section{Conflicts of Interest}

The authors declare no conflicts of interest regarding the publication of this paper.

\section{References}

[1] Kargarzadeh, H., Mariano, M., Huang, J., Lin, N., Ahmad, I., Dufresne, A. and Thomas, S. (2017) Recent Developments on Nanocellulose Reinforced Polymer Nanocomposites: A Review. Polymer, 132, 368-393. https://doi.org/10.1016/j.polymer.2017.09.043

[2] Wang, N., Ding, E. and Cheng, R. (2007) Thermal Degradation Behaviors of Spherical Cellulose Nanocrystals with Sulfate Groups. Polymer, 48, 3486-3493. https://doi.org/10.1016/j.polymer.2007.03.062

[3] Bondeson, D., Mathew, A. and Oksman, K. (2006) Optimization of the Isolation of Nanocrystals from Microcrystalline Cellulose by Acid Hydrolysis. Cellulose, 13, 171. https://doi.org/10.1007/s10570-006-9061-4 
[4] Li, B., Xu, W., Kronlund, D., Määttänen, A., Liu, J., Smått, J.-H., Peltonen, J., Willför, S., Mu, X. and Xu, C. (2015) Cellulose Nanocrystals Prepared via Formic Acid Hydrolysis Followed by TEMPO-Mediated Oxidation. Carbohydrate Polymers, 133, 605-612. https://doi.org/10.1016/j.carbpol.2015.07.033

[5] Melo, A.R.A., da Silva, E.O., Menezes, L.R. and Tavares, M.I.B. (2018) The Effect of Modified Cellulose Particles on Morphology and Properties Ethylene Vinyl Acetate Copolymer. Polymer Testing, 68, 333-339. https://doi.org/10.1016/j.polymertesting.2018.04.012

[6] Camarero Espinosa, S., Kuhnt, T., Foster, E.J. and Weder, C. (2013) Isolation of Thermally Stable Cellulose Nanocrystals by Phosphoric Acid Hydrolysis. Biomacromolecules, 14, 1223-1230. https://doi.org/10.1021/bm400219u

[7] Chen, L., Zhu, J., Baez, C., Kitin, P. and Elder, T. (2016) Highly Thermal-Stable and Functional Cellulose Nanocrystals and Nanofibrils Produced Using Fully Recyclable Organic Acids. Green Chemistry, 18, 3835-3843. https://doi.org/10.1039/C6GC00687F

[8] Xu, W.-J., Zhai, J.-W., Cui, Q., Liu, J.-Z., Luo, M., Fu, Y.-J. and Zu, Y.-G. (2016) Ultra-Turrax Based Ultrasound-Assisted Extraction of Five Organic Acids from Honeysuckle (Lonicera japonica Thunb.) and Optimization of Extraction Process. Separation and Purification Technology, 166, 73-82. https://doi.org/10.1016/j.seppur.2016.04.003

[9] Liu, Y., Wang, H., Yu, G., Yu, Q., Li, B. and Mu, X. (2014) A Novel Approach for the Preparation of Nanocrystalline Cellulose by Using Phosphotungstic Acid. Carbohydrate Polymers, 110, 415-422. https://doi.org/10.1016/j.carbpol.2014.04.040

[10] Lu, Q., Cai, Z., Lin, F., Tang, L., Wang, S. and Huang, B. (2016) Extraction of Cellulose Nanocrystals with a High Yield of $88 \%$ by Simultaneous Mechanochemical Activation and Phosphotungstic Acid Hydrolysis. ACS Sustainable Chemistry \& Engineering, 4, 2165-2172. https://doi.org/10.1021/acssuschemeng.5b01620

[11] Boujemaoui, A., Mongkhontreerat, S., Malmström, E. and Carlmark, A. (2015) Preparation and Characterization of Functionalized Cellulose Nanocrystals. Carbohydrate Polymers, 115, 457-464. https://doi.org/10.1016/j.carbpol.2014.08.110

[12] Jia, C., Chen, L., Shao, Z., Agarwal, U.P., Hu, L. and Zhu, J.Y. (2017) Using a Fully Recyclable Dicarboxylic Acid for Producing Dispersible and Thermally Stable Cellulose Nanomaterials from Different Cellulosic Sources. Cellulose, 24, 2483-2498. https://doi.org/10.1007/s10570-017-1277-y

[13] Li, D., Henschen, J. and Ek, M. (2017) Esterification and Hydrolysis of Cellulose Using Oxalic Acid Dihydrate in a Solvent-Free Reaction Suitable for Preparation of Surface-Functionalised Cellulose Nanocrystals with High Yield. Green Chemistry, 19, 5564-5567. https://doi.org/10.1039/C7GC02489D

[14] Tang, L., Huang, B., Lu, Q., Wang, S., Ou, W., Lin, W. and Chen, X. (2013) Ultrasonication-Assisted Manufacture of Cellulose Nanocrystals Esterified with Acetic Acid. Bioresource Technology, 127, 100-105. https://doi.org/10.1016/j.biortech.2012.09.133

[15] Kargarzadeh, H., Ahmad, I., Abdullah, I., Dufresne, A., Zainudin, S.Y. and Sheltami, R.M. (2012) Effects of Hydrolysis Conditions on the Morphology, Crystallinity and Thermal Stability of Cellulose Nanocrystals Extracted from Kenaf Bast Fibers. Cellulose, 19, 855-866. https://doi.org/10.1007/s10570-012-9684-6

[16] Wang, Q.Q., Zhao, X. and Zhu, J.Y. (2014) Kinetics of Strong Acid Hydrolysis of a Bleached Kraft Pulp for Producing Cellulose Nanocrystals (CNCs). Industrial \& Engineering Chemistry Research, 53, 11007-11014. 
https://doi.org/10.1021/ie501672m

[17] Hamad, W.Y. and Hu, T.Q. (2010) Structure-Process-Yield Interrelations in Nanocrystalline Cellulose Extraction. The Canadian Journal of Chemical Engineering, $8 \mathbf{8}$, 392-402. https://doi.org/10.1002/cjce.20298

[18] Wang, Q.Q., Zhu, J.Y., Reiner, R.S., Verrill, S.P., Baxa, U. and McNeil, S.E. (2012) Cellulose Loss in Cellulose Nanocrystal (CNC) Production: Recovery and Characterization of Cellulosic Solid Residues (CSR) and CNCApproaching Zero. Cellulose, 19, 2033-2047. https://doi.org/10.1007/s10570-012-9765-6

[19] Aguayo, M., Fernández Pérez, A., Reyes, G., Oviedo, C., Gacitúa, W., Gonzalez, R. and Uyarte, O. (2018) Isolation and Characterization of Cellulose Nanocrystals from Rejected Fibers Originated in the Kraft Pulping Process. Polymers, 10, 1145. https://doi.org/10.3390/polym10101145

[20] Chen, L., Wang, Q., Hirth, K., Baez, C., Agarwal, U.P. and Zhu, J. (2015) Tailoring the Yield and Characteristics of Wood Cellulose Nanocrystals (CNC) Using Concentrated Acid Hydrolysis. Cellulose, 22, 1753-1762.

https://doi.org/10.1007/s10570-015-0615-1

[21] Grishkewich, N., Mohammed, N., Tang, J. and Tam, K.C. (2017) Recent Advances in the Application of Cellulose Nanocrystals. Current Opinion in Colloid \& Interface Science, 29, 32-45. https://doi.org/10.1016/j.cocis.2017.01.005

[22] Arputharaj, A., Nadanathangam, V. and Shukla, S.R. (2017) A Simple and Efficient Protocol to Develop Durable Multifunctional Property to Cellulosic Materials Using in Situ Generated Nano-ZnO. Cellulose, 24, 3399-3410. https://doi.org/10.1007/s10570-017-1335-5

[23] Jia, M., Zhang, X., Weng, J., Zhang, J. and Zhang, M. (2019) Protective Coating of Paper Works: ZnO/Cellulose Nanocrystal Composites and Analytical Characterization. Journal of Cultural Heritage, 38, 64-74. https://doi.org/10.1016/j.culher.2019.02.006

[24] Khosravian, S., Montazer, M., Malek, R.M. and Harifi, T. (2015) In Situ Synthesis of Nano ZnO on Starch Sized Cotton Introducing Nano Photo Active Fabric Optimized with Response Surface Methodology. Carbohydrate Polymers, 132, 126-133. https://doi.org/10.1016/j.carbpol.2015.05.085

[25] Azizi, S., Ahmad, M., Mahdavi, M. and Abdolmohammadi, S. (2013) Preparation, Characterization and Antimicrobial Activities of $\mathrm{ZnO}$ Nanoparticles/Cellulose $\mathrm{Na}$ nocrystal Nanocomposites. BioResources, 8, 1841-1851. https://doi.org/10.15376/biores.8.2.1841-1851

[26] Azizi, S., Ahmad, M.B., Ibrahim, N.A., Hussein, M.Z. and Namvar, F. (2014) Cellulose Nanocrystals/ZnO as a Bifunctional Reinforcing Nanocomposite for Poly (Vinyl Alcohol)/Chitosan Blend Films: Fabrication, Characterization and Properties. International Journal of Molecular Sciences, 15, 11040-11053. https://doi.org/10.3390/ijms150611040

[27] Anvzlovar, A., Orel, Z.C., Kogej, K. and Zigon, M. (2012) Polyol-Mediated Synthesis of Zinc Oxide Nanorods and Nanocomposites with Poly (Methyl Methacrylate). Journal of Nanomaterials, 2012, Article ID: 760872.

[28] Jia, W., Dang, S., Liu, H., Zhang, Z., Yu, C., Liu, X. and Xu, B. (2012) Evidence of the Formation Mechanism of $\mathrm{ZnO}$ in Aqueous Solution. Materials Letters, 82, 99-101. https://doi.org/10.1016/j.matlet.2012.05.013

[29] Li, X., Zhang, X., Li, L., Huang, L., Zhang, W. and Ye, J. (2016) Preparation of Nano-ZnO/Regenerated Cellulose Composite Particles via Co-Gelation and Low-Temperature Hydrothermal Synthesis. Materials Letters, 175, 122-125. 
https://doi.org/10.1016/j.matlet.2016.04.012

[30] Znaidi, L. (2010) Sol-Gel-Deposited ZnO Thin Films: A Review. Materials Science and Engineering. B, 174, 18-30. https://doi.org/10.1016/j.mseb.2010.07.001

[31] Eyholzer, C., Bordeanu, N., Lopez-Suevos, F., Rentsch, D., Zimmermann, T. and Oksman, K. (2010) Preparation and Characterization of Water-Redispersible Nanofibrillated Cellulose in Powder Form. Cellulose, 17, 19-30. https://doi.org/10.1007/s10570-009-9372-3

[32] Jin, E., Guo, J., Yang, F., Zhu, Y., Song, J., Jin, Y. and Rojas, O. J. (2016) On the Polymorphic and Morphological Changes of Cellulose Nanocrystals (CNC-I) Upon Mercerization and Conversion to CNC-II. Carbohydrate Polymers, 143, 327-335. https://doi.org/10.1016/j.carbpol.2016.01.048

[33] Khoshkava, V. and Kamal, M. (2014) Effect of Drying Conditions on Cellulose Nanocrystal (CNC) Agglomerate Porosity and Dispersibility in Polymer Nanocomposites. Powder Technology, 261, 288-298.

https://doi.org/10.1016/j.powtec.2014.04.016

[34] Sèbe, G., Ham-Pichavant, F., Ibarboure, E., Koffi, A.L.C. and Tingaut, P. (2012) Supramolecular Structure Characterization of Cellulose II Nanowhiskers Produced by Acid Hydrolysis of Cellulose I Substrates. BioMacromolecules, 13, 570-578. https://doi.org/10.1021/bm201777j

[35] Jayachandiran, J., Vajravijayan, S., Nandhagopal, N., Gunasekaran, K. and Nedumaran, D. (2019) Fabrication and Characterization of ZnO Incorporated Cellulose Microfiber Film: Structural, Morphological and Functional Investigations. Journal of Materials Science: Materials in Electronics, 30, 6037-6049. https://doi.org/10.1007/s10854-019-00904-1

[36] Singh, A., Viswanath, V. and Janu, V. (2009) Synthesis, Effect of Capping Agents, Structural, Optical and Photoluminescence Properties of $\mathrm{ZnO}$ Nanoparticles. Journal of Luminescence, 129, 874-878. https://doi.org/10.1016/j.jlumin.2009.03.027

[37] Nam, S., French, A.D., Condon, B.D. and Concha, M. (2016) Segal Crystallinity Index Revisited by the Simulation of X-Ray Diffraction Patterns of Cotton Cellulose I $\beta$ and Cellulose II. Carbohydrate Polymers, 135, 1-9. https://doi.org/10.1016/j.carbpol.2015.08.035

[38] Ramírez, J.A.Á., Suriano, C.J., Cerrutti, P. and Foresti, M.L. (2014) Surface Esterification of Cellulose Nanofibers by a Simple Organocatalytic Methodology. Carbohydrate Polymers, 114, 416-423. https://doi.org/10.1016/j.carbpol.2014.08.020

[39] Giraldi, T.R., Santos, G.V., Mendonça, V.R., Ribeiro, C. and Weber, I.T. (2011) Annealing Effects on the Photocatalytic Activity of $\mathrm{ZnO}$ Nanoparticles. Journal of Nanoscience and Nanotechnology, 11, 3635-3640.

https://doi.org/10.1166/jnn.2011.3801

[40] Grunin, L.Y., Grunin, Y.B., Nikolskaya, E.A., Sheveleva, N.N. and Nikolaev, I.A. (2017) An NMR Relaxation and Spin Diffusion Study of Cellulose Structure during Water Adsorption. Biophysics (Russian Federation), 62, 198-206. https://doi.org/10.1134/S0006350917020087

[41] Mackay, A.L., Tepfer, M., Taylor, I.E.P. and Volke, F. (1985) Proton Nuclear Magnetic Resonance Moment and Relaxation Study of Cellulose Morphology. Macromolecules, 18, 1124-1129. https://doi.org/10.1021/ma00148a013

[42] Papon, A., Saalwächter, K., Schäler, K., Guy, L., Lequeux, F. and Montes, H. (2011) Low-Field NMR Investigations of Nanocomposites: Polymer Dynamics and Network Effects. Macromolecules, 44, 913-922. https://doi.org/10.1021/ma102486x 